\title{
IMPLICIT DIFFERENTIAL EQUATIONS FROM THE SINGULARITY THEORY VIEWPOINT
}

\author{
J. W. BRUCE and F. TARI \\ Department of Pure Mathematics, The University of Liverpool \\ PO Box 147, Liverpool L69 3BX, England \\ E-mail: jwbruce@liverpool.ac.uk
}

1. Introduction. An implicit differential equation (IDE) is any equation of the form

$$
F(x, y, d y / d x)=0
$$

where $F$ is some smooth function of 3 variables. The term implicit is used to contrast an equation of this general form with one we can write as

$$
d y / d x=g(x, y)
$$

where the derivative is given explicitly as a function of the variables $x$ and $y$. The latter can be solved (numerically) using the Euler line method for example, and there is generally a single solution curve through each point of the plane. A more convenient form of the above explicit equation is

$$
a(x, y) d y+b(x, y) d x=0 .
$$

Geometrically this equation determines a line field, that is a line is assigned to each point in the plane, with the specified slope, and a solution is a smooth curve with the property that at each of its points it is tangent to the given line. Of course the generally isolated points in the plane where the functions $a$ and $b$ both vanish are special, and are referred to as singular points of the ordinary differential equation (ODE). An implicit differential equation, on the other hand, will generally determine several lines through a given point $(x, y)$ in the plane, and hence several solution curves there. It can roughly be thought of as the superposition of a number of ODE's and singularities can arise in three ways. There are those points where two or more of the lines coincide, those where one of the constituent ODE's has a singularity, and points where both occur.

1991 Mathematics Subject Classification: 58F, 34C.

Research of the second author supported by the Esprit grant VIVA.

The paper is in final form and no version of it will be published elsewhere. 
Implicit differential equations occur in a number of branches of mathematics, and in particular in the differential geometry of surfaces in 3-space.

EXAMPLE 1.1 (From differential geometry). (a) Let $X$ be smooth surface in Euclidean 3 -space. At a hyperbolic point on $X$ there are two directions, called asymptotic directions, in which the sectional curvature is zero. (These are also the tangents to the two branches of the double point of the curve of intersection of the surface and its tangent plane.) At a parabolic point these directions coincide, and there are no asymptotic directions at elliptic points. The result is a bivalued directional field on the non-elliptic part of the surface whose integral curves are the so-called asymptotic curves.

Suppose that $X$ is given locally in Monge form $(x, y, h(x, y))$. The asymptotic directions at $(x, y, h(x, y))$ are then the solution curves of the IDE

$$
h_{y y} d y^{2}+2 h_{x y} d x d y+h_{x x} d x^{2}=0 .
$$

(b) Another classical example is provided by the principal directions field on $X$. At a non-umbilic point there are two orthogonal directions corresponding to extrema of the sectional curvature, at an umbilic however all directions are principal. So away from umbilics the resulting field of directions integrates to mutually orthogonal lines of curvatures. To understand their structure at an umbilic we need to write down the corresponding IDE.

If we take the surface in Monge form at the umbilic then $h(x, y)=\frac{\kappa}{2}\left(x^{2}+y^{2}\right)+C(x, y)$ where $\kappa$ is the sectional curvature at the umbilic and the function $C$ is 2 -flat. The IDE determining the principal directions is then of the form

$$
-\left(\partial^{2} C / \partial x \partial y\right) d y^{2}+\left(\partial^{2} C / \partial y^{2}-\partial^{2} C / \partial x^{2}\right) d y d x+\left(\partial^{2} C / \partial x \partial y\right) d x^{2}=0
$$

The determination of the topological structure of the lines of curvatures at umbilics has a long history; see for example [5], [10]. (See Figure 1)

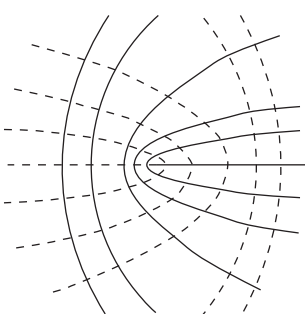

Lemon

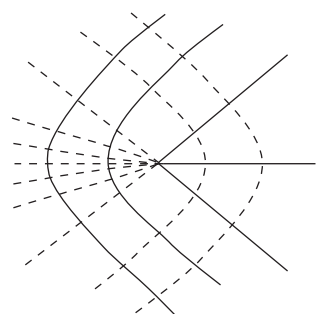

Monstar

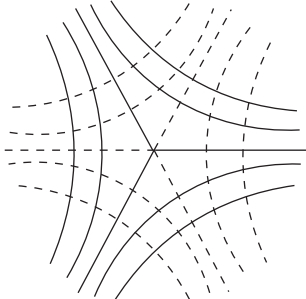

Star

Fig. 1. Lines of curvatures at an umbilic point

EXAMPLE 1.2 (General eigenvalue problem [5], [6]). Consider a general 2 by 2 matrix $A=\left(\begin{array}{ll}a & b \\ c & d\end{array}\right)$. The characteristic polynomial of $A$ is $t^{2}-(a+d) t+(a d-b c)$. Thus there are two distinct real eigenvalues if $\delta=(a-d)^{2}+4 b c>0$, a real repeated eigenvalue if $\delta=0$ and complex eigenvalues if $\delta<0$.

Suppose that we have a smooth map germ $\phi: \mathbb{R}^{2}, 0 \rightarrow M_{2}$, where $M_{2}$ is the space of 2 by 2 matrices. If $\Delta \subset M_{2}$ is given by $\delta=0$ then $\phi$ determines a bivalued field on the region $\delta \circ \phi>0$ with boundary $\phi^{-1}(\Delta)$; at a point $(x, y) \in \mathbb{R}^{2}$ we consider the 
translate of the two eigenspaces determined by $\phi(x, y)$ and the map $\phi$ determines an IDE. Eliminating $\lambda$ from $A\left(\begin{array}{l}x \\ y\end{array}\right)=\lambda\left(\begin{array}{l}x \\ y\end{array}\right)$ we obtain $b y^{2}+(a-d) x y-c x^{2}=0$ and viewing $a, b, c, d$ as the four components of $\phi$ yields

$$
b d y^{2}+(a-d) d x d y-c d x^{2}=0
$$

Naturally one can consider the case where we have a smooth map $\phi$ with target $S M_{2}$ the space of symmetric 2 by 2 matrices. Thus $b$ and $c$ coincide and we obtain a IDE

$$
b d y^{2}+(a-d) d x d y-b d x^{2}=0 .
$$

That the sum of the $d y^{2}$ and $d x^{2}$ coefficients is zero is equivalent to the fact that the eigenspaces are orthogonal. Note that in this case we have two distinct directions except at those points where $b=(a-d)=0$. These equations generally determine a collection of isolated points in the plane.

ExAmple 1.3 (Stretch and compression). Let $M$ and $N$ be two Riemannian manifolds of dimension 2 and let $\Phi: M \rightarrow N$ be a smooth map. Suppose that $\Phi$ is the germ of a diffeomorphism at $x \in M$. Then $d \Phi_{x}: T_{x} M \rightarrow T_{\Phi(x)} N$ is an isomorphism. For $v \in T_{x} M$ a unit vector we can consider the length of the image of $v$ under the derivative of $\Phi$, $\left\|d \Phi_{x}(v)\right\|_{\Phi(x)}$ and seek those $v$ for which this is maximized $/$ minimized. If we choose an orthonormal basis for $T_{x} M$ and $T_{\Phi(x)} N$ and write $d \Phi_{x}=\left(\begin{array}{ll}a & b \\ c & d\end{array}\right)$ with respect to these bases, we are maximizing/minimizing $(a \cos \theta+b \sin \theta)^{2}+(c \cos \theta+d \sin \theta)^{2}$ with respect to $\theta$. Differentiating this expression yields

$$
-\left(a^{2}+c^{2}\right) \cos \theta \sin \theta+2(a b+c d)\left(\cos ^{2} \theta-\sin ^{2} \theta\right)+\left(b^{2}+d^{2}\right) \cos \theta \sin \theta=0 .
$$

Therefore

$$
\left\{\left(b^{2}+d^{2}\right)-\left(a^{2}+c^{2}\right)\right\} \sin 2 \theta+2(a b+c d) \cos 2 \theta=0 .
$$

So we obtain two orthogonal directions in which we have an extremum, given by the IDE

$$
(a b+c d) d x^{2}+\left(b^{2}+d^{2}-\left(a^{2}+c^{2}\right)\right) d x d y-(a b+c d) d y^{2}=0 .
$$

Geometrically these are the axes of the ellipse in $T_{\Phi(x)} N$ which is the image of the unit circle in $T_{x} M$. The coefficients vanish when $a b+c d=b^{2}+d^{2}-\left(a^{2}+c^{2}\right)=0$. This occurs when $c= \pm b$ and $a=\mp d$, so $d \Phi_{x}$ is of the form reflexion in a line plus dilation or rotation plus dilation, as one might expect; in other words when the image of the unit circle is also a circle. (Note that we only need a conformal structure on $M$ and $N$.)

We remark that when $M$ is a surface in 3-space, $N=S^{2}$, and $\Phi$ is the Gauss map then the vectors above are the principal directions of $M$.

EXAMPLE 1.4 (Swimmer in the flow of a fluid). This example is taken from [12] (see $[9])$. In the plane of the swimmer the flow of water has velocity $(x, \beta y), \beta>2$, at $(x, y)$. The swimmer, in standing water, can swim in any direction with unit speed. The question is where can the swimmer wind up when starting from a given point of the plane. The inequality $x^{2}+\beta^{2} y^{2}>1$ gives the so-called steep domain at each point of which the direction of possible velocities of the swimmer forms an angle less than $180^{\circ}$. The sides of this angle are called the limiting directions at this point. So in the steep domain there is a bivalued field of limiting directions. The integral curves of this field are called the 
limiting lines and are precisely those of the equation

$$
(x d y-\beta y d x)^{2}\left(x^{2}+\beta^{2} y^{2}-1\right)=(x d x+\beta y d y)^{2} .
$$

From a given point $S$ in the steep domain the boundary of the region which can be reached from $S$ is given by some subset of the integral curves through $S$.

The aim of much of the work on IDE's is to obtain normal forms for the simplest of their 'singularities'. As with ODE's (singularities of vector fields) there are three types of question one can ask. Firstly one can seek smooth normal forms. These normally involve moduli (even functional moduli). Then one can seek normal forms up to homeomorphism. Finally as one moves to non-generic situations one can seek an understanding of the way in which more complicated singularities break up on perturbation. In each case we are only concerned with the local theory, i.e. that concerning germs. In this paper we start by considering two geometrical constructions for lifting an IDE to an ODE on a suitable associated surface $(\S 2,3)$. We then consider the formal reductions of a certain class of IDE's, the binary differential equations (BDE's, see $\S 4$ ), and review the classification of Davydov of singularities of such systems ( 55 ). Finally we discuss the formal and topological classification of BDE's when the so-called discriminant has a Morse singularity.

2. Disentangling. Singularity theory has proved to be an important tool in the study of IDE's, although their study has a long history. The key to many approaches to this subject is some method of disentangling the IDE's into a single ODE on a more complicated space. Given an IDE $F(x, y, d y, d x)=0$ one obvious way to get started is to consider the projective tangent bundle to the plane, which is simply $\mathbb{R}^{2} \times \mathbb{R} P^{1}$, and the subset

$$
\{(x, y,[p: q]): F(x, y, p, q)=0\} .
$$

It is often more convenient to choose an affine chart in $\mathbb{R} P^{1}$ and consider the subset of $(x, y, p)$ space given by $F(x, y, p, 1)=0$. By Sard's theorem this will generally be a smooth surface say $M$. There is a natural projection

$$
\pi: M \rightarrow \mathbb{R}^{2}, \quad(x, y, p) \mapsto(x, y),
$$

and one lifts the IDE on the target to an ODE on the source. At each point $(x, y, p)$ of $M$ we choose a direction tangent to $M$ which projects down to a line through $(x, y)$ with slope $p$. This can be done quite explicitly: the vector field

$$
\xi=F_{p} \partial / \partial x+p F_{p} \partial / \partial y-\left(F_{x}+p F_{y}\right) \partial / \partial p
$$

is clearly tangent to $M$, and projects, at each point $(x, y, p)$ to a vector through $(x, y)$ with slope $p$. This lift $\xi$ determines an ODE on $M$ which we can then analyse.

Points of $M$ where the projection $\pi$ is not a local diffeomorphism are of especial interest. These are points where we cannot solve $F=0$ for $p$, that is cannot reduce locally to an ODE. They are also the points where two or more of the directions coincide. This set of critical points of $\pi$ is called the criminant and consists of points where the tangent plane to the surface $M$ is vertical. The projection of the criminant is the discriminant of the equation. A result of Whitney [14] states that for an open dense set of functions 
$F$ the projection $\pi$ is either a local diffeomorphism, a fold or a cusp map. For the fold singularity, one can choose local coordinates in $M$ and $\mathbb{R}^{2}$ for which $\pi$ has the form $\left(u, v^{2}\right)$. The cusp has normal form $\left(u, v^{3}+u v\right)$.

So at most points the singularities will be fold points, and the set of critical values locally a smooth curve separating points of the plane where there are two solution curves from points where there are none. Indeed the condition for a fold is simply $F=F_{p}=0$, $F_{p p} \neq 0$. On this criminant there will also be special points where the lifted field has a zero. In terms of the lift constructed above these are points satisfying

$$
F=F_{p}=F_{x}+p F_{y}=0 .
$$

Note that all zeros of the lift $\xi$ must lie on the criminant.

In all of the examples given above there were at most two directions at each point of the plane. IDE's of this form are called binary differential equations, BDE's, and are of especial interest. In particular locally at least we shall be dealing with a BDE when the projection from the surface $F=0$ has a fold singularity. Using the Preparation Theorem [2] we can always write such a BDE in the form

$$
a(x, y) d y^{2}+2 b(x, y) d x d y+c(x, y) d x^{2}=0
$$

where $a, b, c$ are smooth functions. Indeed in the case of a fold singularity we may suppose that $a(x, y)$ is identically 1 , but it is useful to consider the more general form in what follows. The discriminant in case (2) is simply given by the equation $b^{2}-a c=0$. When the functions $a, b, c$ all vanish at the origin the discriminant becomes singular. This occurs in generic 1-parameter family of BDEs, but can be a stable feature in special situations, like that of the principal directions field at an umbilic point on a smooth surface, or the eigenvalue problem discussed above in the case when $\phi(0)$ is the identity. See [5], [10] and Section 7 below.

3. Legendre transformation. Another method of disentangling IDE's worthy of mention is the Legendre transformation. This is an automorphism of $(x, y, p)$-space given by setting

$$
X=p, \quad Y=x p-y, \quad P=x
$$

and clearly defines a smooth involution on $\mathbb{R}^{3}$. Each $\operatorname{IDE} F(x, y, p)=0$ yields, via this transform, a new IDE defined by $G(X, Y, P)=F(P, X P-Y, X)=0$. (See [1], p. 19.) The advantage of this approach is that while the point $(x, y, p)$ may lie on the criminant of $G=0$ the corresponding point of $F=0$ may not, that is this new equation may be solvable for $P$ at the relevant point.

At a fold singularity of the projection $\pi$ away from singularities of the lifted field, an integral curve $(X(t), Y(t))$ of $G$ yields a solution curve of the original equation $F=$ 0 parametrised as $(x(t), y(t))=\left(Y^{\prime}(t), X(t) Y^{\prime}(t)-Y(t)\right)$. The latter two components give the gradient and intercept for the tangent line to the curve $(X(t), Y(t))$, i.e. this is the dual, [3]. It is not difficult to check that inflexions on the solutio n curves of $G=0$ correspond exactly to points on the criminant $F=F_{p}=0$, and we have ordinary inflexions precisely when $F_{p p} \neq 0$, i.e. we have an ordinary fold point. Since the dual of an ordinary inflexion is a cusp this shows that integral curves along the discriminant 
generally have cusps. (This is also the result proved by Dara [7], and on page 27 of [1].) When the projection $\pi$ has a cusp one can show, using these ideas, that the 1-parameter family of integral curves near such a point when spread out into three space by using the parameter as a third coordinate, form a swallowtail and the individual integral curves are obtained by slicing this swallowtail by a family of generic planes. However, as shown in [3], it is not possible to exhibit a model, up to smooth changes of coordinates, of the solution curves at the cusp point. Davydov subsequently showed that this is so even if we allow topological changes of coordinates, [9]. (There are functional moduli.)

The Legendre transformation was also used by Darboux [8] in his investigation of the configuration of lines of curvatures at umbilic points. He considered only the initial terms in the coefficients of the BDE governing these lines (Example 1). The Legendre transformation takes this linear BDE to an ordinary differential equation. One can explicitly solve this equation and take the duals of the resulting integral curves. Darboux found in this way the configurations in Figure 1. Of course one needs to know that these equations are '1-determined' for this to be relevant. (See Section 6 below.)

4. Normal forms for BDEs with smooth discriminant. In this section we shall attempt to reduce BDE's to normal form, and start by reviewing the situation for an ODE. The theory of formal reduction of vector fields to a normal form was developed by Poincaré. His aim was to transform a non-resonant vector field to its linear part at a singular point by a formal diffeomorphism. Poincaré's method consists of annihilating successively all terms of degree $k(k \geq 2)$ in the equation. Although this process is not always convergent it is nevertheless "a powerful device in the study of differential equations: a few initial terms of the series often give significant information on the behaviour of solutions, which is sufficient for the construction of the phase portrait" [1], Chapter 5 .

The convergence problem is a good deal more subtle (again see [1], chapter 5). A theorem of Siegel asserts that if the eigenvalues of the linear part of the vector field are of type $(C, \mu)$ (a rather technical condition satisfied by almost all linear ODE's) then the process of sucessive annihilation of higher terms converges. As a consequence, the germ of a singular point of a generic vector field in the plane can be reduced to one of the normal forms

$$
\begin{array}{ll}
\text { Saddle } & \xi_{1}=x \partial / \partial x+\alpha y \partial / \partial y, \\
\text { Node } & \xi_{2}=x \partial / \partial x+\alpha y \partial / \partial y, \\
\text { Focus } & \xi_{3}=(x-\alpha y) \partial / \partial x+(\alpha x+y) \partial / \partial y,
\end{array}
$$

where $\alpha$ is negative for a saddle, and positive for a node.

When allowing topological changes of coordinates one can eliminate the parameter $\alpha$ and obtain simple normal forms:

$$
\begin{aligned}
& x \partial / \partial x-2 y \partial / \partial y, \\
& x \partial / \partial x+2 y \partial / \partial y, \\
& (x-y) \partial / \partial x+(x+y) \partial / \partial y .
\end{aligned}
$$

We now consider binary differential equations (2) where $a, b, c$ are smooth functions not all vanishing $(0,0)$, and start by considering the effect of a linear change of coordinates 
on such a BDE. Indeed we consider the effect on the constant terms of the coefficients $a, b$ and $c$. So set

$$
\begin{aligned}
& x=\alpha X+\beta Y \\
& y=\gamma X+\delta Y
\end{aligned} \quad \text { so that } \quad \begin{aligned}
& d x=\alpha d X+\beta d Y \\
& d y=\gamma d X+\delta d Y
\end{aligned}
$$

and denoting constant terms of (2) by $A, 2 B, C$ respectively, the constant terms of the new BDE are as follows:

$$
\begin{aligned}
& A^{\prime}=A \beta^{2}+2 B \beta \delta+C \delta^{2}, \\
& B^{\prime}=A \gamma \delta+B(\alpha \delta+\beta \gamma)+C \alpha \beta, \\
& C^{\prime}=A \gamma^{2}+2 B \alpha \gamma+C \alpha^{2} .
\end{aligned}
$$

These new coefficients $A^{\prime}, B^{\prime}, C^{\prime}$ then can be written

$$
\left(\begin{array}{ll}
\beta & \delta \\
\alpha & \gamma
\end{array}\right)\left(\begin{array}{ll}
A & B \\
B & C
\end{array}\right)\left(\begin{array}{ll}
\beta & \alpha \\
\delta & \gamma
\end{array}\right)=\left(\begin{array}{ll}
A^{\prime} & B^{\prime} \\
B^{\prime} & C^{\prime}
\end{array}\right)
$$

Now symmetric matrices up to this notion of equivalence have six equivalence classes, namely

$$
\left(\begin{array}{ll}
1 & 0 \\
0 & 1
\end{array}\right),\left(\begin{array}{cc}
1 & 0 \\
0 & -1
\end{array}\right),\left(\begin{array}{cc}
-1 & 0 \\
0 & -1
\end{array}\right),\left(\begin{array}{ll}
1 & 0 \\
0 & 0
\end{array}\right),\left(\begin{array}{cc}
-1 & 0 \\
0 & 0
\end{array}\right) \text { and }\left(\begin{array}{ll}
0 & 0 \\
0 & 0
\end{array}\right) .
$$

So we have the following possibilities for the constant parts of the BDE:

$$
d y^{2}+d x^{2}, \quad d y^{2}-d x^{2}, \quad d y^{2}, \quad 0
$$

since we can multiply by non-zero constants. We shall largely be considering changes of coordinates of the form

$$
x=X+p(X, Y), \quad y=Y+q(X, Y),
$$

where $p, q$ (resp. $r$ ) are homogeneous of degree $k \geq 2$. So

$$
d x=\left(1+p_{X}\right) d X+p_{Y} d Y, \quad d y=q_{X} d X+\left(1+q_{Y}\right) d Y .
$$

We are also allowed to multiply our equation by any function which does not vanish at the origin, and will use those of the form $1+r(X, Y)$ where $r$ is homogeneous of degree $k \geq 1$.We shall simplify the coefficients $a, b, c$ of our BDE inductively, and to do this it is crucial what form the initial terms take.

The first two cases we shall consider are rather trivial but illustrate the ideas involved.

4.1. The initial form $d y^{2}+d x^{2}$. Suppose we have reduced the $k$-jet of the BDE to the form

$$
\left(1+a_{k}\right) d y^{2}+2 b_{k} d x d y+\left(1+c_{k}\right) d x^{2}
$$

where $a_{k}, b_{k}, c_{k}$ are homogeneous of degree $k$. If $p, q$ are homogeneous of degree $k+1$, and $r$ is homogeneous of degree $k$, then the $k$-jet of the transformed BDE is obtained by multiplying out

$$
\begin{aligned}
(1+r)\left\{( 1 + a _ { k } ) \left(q_{X} d X+(1+\right.\right. & \left.\left.q_{Y}\right) d Y\right)^{2} \\
& +2 b_{k}\left(q_{X} d X+\left(1+q_{Y}\right) d Y\right)\left(\left(1+p_{X}\right) d X+p_{Y} d Y\right) \\
& \left.+\left(1+c_{k}\right)\left(\left(1+p_{X}\right) d X+p_{Y} d Y\right)^{2}\right\} .
\end{aligned}
$$


Note that we only need to replace $x$ and $y$ by $X$ and $Y$ in the expressions $a_{k}, b_{k}, c_{k}$ since they are of degree $\geq 1$, and the new terms would be of degree $\geq k+1$. We obtain the homogeneous parts of degree $k$ of the coefficient of the new BDE:

$$
\begin{aligned}
& A_{k}=a_{k}+2 p_{Y}+r, \\
& B_{k}=b_{k}+p_{X}+q_{Y}, \\
& C_{k}=c_{k} y+2 q_{X}+r .
\end{aligned}
$$

If $H^{k}$ denotes the space of homogeneous polynomials of degree $k$ in the variables $x, y$ we claim that the linear map

$$
\begin{aligned}
H^{k+1} \oplus H^{k+1} \oplus H^{k} & \rightarrow H^{k} \oplus H^{k} \oplus H^{k}, \\
(p, q, r) & \mapsto\left(2 p_{Y}+r, p_{X}+q_{Y}, 2 q_{X}+r\right),
\end{aligned}
$$

is surjective. It then follows that we can choose $p, q, r$ so that $A_{k}=B_{k}=C_{k}=0$. To prove surjectivity suppose that $(p, q, r)$ lies in the kernel of this map. Then $p_{X}+q_{Y}=$ $p_{Y}-q_{X}=0$, and conversely these two conditions imply that we can find an $r$ for which the linear map is zero.

Write $p=\sum_{i=0}^{k+1} p_{i} x^{k+1-i} y^{i}$ and $q i=\sum_{i=0}^{k+1} q_{i} x^{k+1-i} y^{i}$. The two conditions imply that

$$
\begin{aligned}
& \sum_{i=0}^{k+1}(k+1-i) p_{i} x^{k-i} y^{i}+\sum_{i=0}^{k+1} i q_{i} x^{k+1-i} y^{i-1}=0, \\
& \sum_{i=0}^{k+1} i p_{i} x^{k+1-i} y^{i-1}+\sum_{i=0}^{k+1}(k+1-i) q_{i} x^{k-i} y^{i}=0 .
\end{aligned}
$$

Equating coefficients we find that

$$
\begin{array}{ll}
(k+1-i) p_{i}+(i+1) q_{i+1}=0, & 0 \leq i \leq k, \\
(i+1) p_{i+1}-(k+1-i) q_{i}=0, & 0 \leq i \leq k,
\end{array}
$$

so that

$$
q_{i+1}=\frac{i-k-1}{i+1} p_{i}=\frac{i+2}{k-i} p_{i+2} .
$$

It is not difficult to see that these equations have two independent solutions by taking $p_{0}=1, p_{1}=0$ and $p_{0}=0, p_{1}=1$. So our linear map has nullity two and hence is surjective. We can therefore deduce the following.

Proposition 4.1. Suppose that the BDE has constant part $d y^{2}+d x^{2}$. Then for any $k \geq 1$ we can change coordinates so that the transformed BDE has $k$-jet $d y^{2}+d x^{2}$.

Similarly we have

Proposition 4.2. Suppose that the BDE has constant part $d y^{2}-d x^{2}$. Then for any $k \geq 1$ we can change coordinates so that the transformed BDE has $k$-jet $d y^{2}-d x^{2}$.

We shall now address the question of convergence of this process of successive annihilation of homogeneous terms of degree $k \geq 2$. The resulting linear equations can be rewritten $p_{X}+q_{Y}=-b_{k}, p_{Y}-q_{X}=-\frac{1}{2}\left(a_{k}-c_{k}\right), r=-c_{k}-2 q_{X}$. The first two equations yield a linear map

$$
L_{k}: H^{k+1} \oplus H^{k+1} \rightarrow H^{k+1} \oplus H^{k+1}, \quad(p, q) \mapsto\left(p_{X}+q_{Y}, p_{Y}-q_{X}\right) .
$$


This is also surjective, in fact if we set $p=\sum_{i=0}^{k+1} p_{i} x^{k+1-i} y^{i}$ and $q=\sum_{i=0}^{k+1} q_{i} x^{k+1-i} y^{i}$, then the restriction of $L_{k}$ to $p_{0}=p_{1}=0$ is an isomorphism. In what follows we shall assume that the change of coordinates satisfies $p_{0}=p_{1}=0$ at each step.

Proposition 4.3. Suppose the BDE has constant part $d y^{2}+d x^{2}$. Then there exists an analytic change of coordinates transforming the BDE to the constant part $d y^{2}+d x^{2}$.

Pr o of. The proof follows that of Siegel's Theorem, we refer to [1], pp. 212-218, for the substantial technicalities involved. We claim that the multiplicative type condition on the eigenvalues ([1], p. 211) can be replaced by the condition

$$
\left\|\bar{L}_{k}^{-1}\right\| \leq C k^{p}
$$

for some positive constant $C$, a positive integer $p$ and any $k \geq 2$. This condition ensures that all the steps of the proof of Siegel's Theorem remain valid. Calculations show that $\left\|\bar{L}_{k}^{-1}\right\| \leq k^{4}$. The process is thus convergent.

Similarly, we have

Proposition 4.4. Suppose the BDE has constant part $d y^{2}-d x^{2}$. Then there exists an analytic change of coordinates transforming the BDE to the constant part $d y^{2}-d x^{2}$.

Of course there is no geometrical content to 4.3 ; in this case there are no integral curves. For 4.4 we simply note that the linear part of the BDE shows that there are two transverse integral curves through the origin, and all we have done is find an analytic diffeomorphism of the plane taking the integral curves of the BDE to the lines $y=$ $\pm x+$ constant .

4.2. The initial form $d y^{2}$. We next consider the linear part of the BDE whose constant term $d y^{2}$ is

$$
\left(1+a_{1} x+a_{2} y\right) d y^{2}+2\left(b_{1} x+b_{2} y\right) d x d y+\left(c_{1} x+c_{2} y\right) d x^{2} .
$$

Multiplying by $\left(1+a_{1} x+a_{2} y\right)^{-1}$ we may suppose $a_{1}=a_{2}=0$. Now applying the change of coordinates $x=\alpha x+\beta Y, y=Y+\gamma Y^{2}+2 \delta X Y+\delta Y^{2}$ we find that the orbits in the space of 1 -jets have representatives

$$
d y^{2}+x d x^{2}, d y^{2}-y d x^{2}, d y^{2} .
$$

(1) The case $d y^{2}+x d x^{2}$. We proceed as for case I. Assume that the $k$-jets of the coefficients of the BDE are $1+a_{k}, 2 b_{k}, x+c_{k}$ respectively with $a_{k}, b_{k}, c_{k}$ homogeneous of degree $k$. A change of coordinates in the form

$$
x=X+p(X, Y), \quad y=Y+q(X, Y),
$$

with $p \in H^{k}$ and $q \in H^{k+1}$ and multiplication by $1+r(X, Y), r \in H^{k}$ transforms the $k$-jet of the coefficients of the BDE to

$$
\left(1+a_{k}+r+2 q_{Y}\right) d Y^{2}+2\left(b_{k}+q_{X}+X_{Y}\right) d X d Y+\left(x+c_{k}+p+X p_{X}\right) d X^{2} .
$$

The process of annihilating terms of degree $k$ produces a linear map

$$
\begin{aligned}
L_{k}: H^{k} \oplus H^{k+1} \oplus H^{k} & \rightarrow H^{k} \oplus H^{k} \oplus H^{k}, \\
(p, q, r) & \mapsto\left(r+2 q_{Y}, p+2 X p_{X}, q_{X}+X p_{Y}\right) .
\end{aligned}
$$


The linear map $L_{k}$ is surjective and furthermore $\bar{L}_{k}=\left.L_{k}\right|_{q_{k+1}=0}$ is an isomorphism (notations as in case I). We shall consider only changes of coordinates in the source that satisfy $q_{k+1}=0$ at every stage, and obtain

Proposition 4.5. Suppose that the BDE has constant part $d y^{2}+x d x^{2}$. Then for any $k \geq 1$ we can change coordinates so that the transformed BDE has $k$-jet $d y^{2}+x d x^{2}$.

The linear map $\bar{L}_{k}$ satisfies $\left\|\bar{L}_{k}^{-1}\right\| \leq 3 k^{2}$. So by the proof of Proposition 4.3 we have:

Proposition 4.6. Suppose the BDE has constant part $d y^{2}+x d x^{2}$. Then there exist an analytic change of coordinates transforming the BDE to the constant part $d y^{2}+x d x^{2}$.

The geometrical import of this result is that at a general point of the criminant we can find a change of coordinates taking the integral curves to the family of cusps $(x, y)=\left(-t^{2}, 2 / 3 t^{3}+c\right)$. This result with relevant references was mentioned in section 2 .

(2) The case $d y^{2}-y d x^{2}$. This case is harder to deal with. If we proceed as before and use a preliminary change of coordinates we can reduce the 2-jet to $d y^{2}-\left(y+\lambda x^{2}\right) d x^{2}$. If we then regard this as a $k$-jet and attempt as before to eliminate all homogeneous monomials at the $k$-jet level we have problems with the $\left(0,0, x^{k}\right)$ term.

To make progress we need some new tools. We shall be using more general changes of coordinates, and take an 'infinitesimal' approach. We therefore need to compute the tangent space to the orbit of a $\operatorname{BDE}(a, b, c)$ under the 'action' of changes of coordinates in the source and multiplication by non-zero functions in the target. (Note that by taking jets we obtain the action of a Lie group on a vector space.)

For a path of diffeomorphisms of the form $h_{t}=(x+t \alpha(x, y), y)$ say, the corresponding tangent vector obtained by differentiating $h_{t} \cdot(a, b, c)$ with respect to $t$ and setting $t=0$ is

$$
X(\alpha)=\left(\alpha a_{x}+2 \alpha_{y} b, \alpha b_{x}+\alpha_{x} b+\alpha_{y} c, \alpha c_{x}+2 \alpha_{x} c\right) .
$$

Similarly, from a path $(x, y+t \beta(x, y))$ we obtain

$$
Y(\alpha)=\left(\alpha a_{y}+2 \alpha_{y} a, \alpha b_{y}+\alpha_{x} a+\alpha_{y} b, \alpha c_{y}+2 \alpha_{x} b\right) .
$$

Multiplication by $(1+t \alpha)$, differentiating and setting $t=0$ gives

$$
Z(\alpha)=(\alpha a, \alpha b, \alpha c) \text {. }
$$

When working at the level of $k$-jets we simply need to take the $k$-jets of these tangent vectors. In fact we need to consider the action of a unipotent subgroup of the jet group [4]. Clearly for that part of the group corresponding to multiplication this means that the 1-jet of the factor is 1 , so the corresponding tangent vectors in the Lie algebra simply consisit of elements $\beta$ in the maximal ideal $\mathcal{M}$. For the changes of coordinates in the source the subgroup of $\mathrm{k}$-jets of diffeomorphisms with 1-jet the identity is certainly unipotent. This gives rise to vectors $X(\alpha), Y(\alpha)$ with $\alpha \in \mathcal{M}^{2}$. We are also allowed, for example, changes of coordinates of the form $(x+t y, y)$ which yields

$$
X(y)=\left(2 b+y a_{x}, c+y b_{x}, y c_{x}\right)
$$

The unipotent group of $k$-jets of this form will be denoted by $U^{(k)}$, and the tangent space to the resulting orbit of $(a, b, c)$ is denoted by $L U^{(k)} \cdot(a, b, c)$. Before we state the required result we need a definition. 
DEFINITION 4.7. Let $(a, b, c)$ be a BDE. We say that this is formally $k$-determined if for any $r \geq k$ any $\operatorname{BDE}(A, B, C)$ with the same $k$-jet as $(a, b, c)$ is equivalent to a $\operatorname{BDE}$ with the same $r$-jet as $(a, b, c)$.

THEOREM 4.8. The BDE $(a, b, c)$ is formally $k$-determined if for all $r \geq k$ the tangent space $L U^{(r)}$. $(a, b, c)$ contains all vectors of the form $\left(\phi_{1}, \phi_{2}, \phi_{3}\right)$ where the $\phi_{j}$ are of degree $\geq k+1$.

(The proof can be adapted in a straighforward way from those in [4].)

We are considering the case $(a, b, c)=\left(1,0,-y-\lambda x^{2}\right)$, and we now need to check that the tangent space contains all terms of degree $\geq 3$. Note that

$$
\begin{aligned}
& X(\alpha)=-\left(0, \alpha_{y}\left(y+\lambda x^{2}\right), 2 \alpha_{x}\left(y+\lambda x^{2}\right)\right), \\
& Y(\alpha)=\left(2 \alpha_{y}, \alpha_{x},-\alpha\right), \\
& Z(\alpha)=\alpha\left(1,0,-y-\lambda x^{2}\right) .
\end{aligned}
$$

It is enough to show that for each $k \geq 3$ all elements of the form $\left(\phi_{1}, \phi_{2}, \phi_{3}\right)$, with the $\phi_{i}$ homogeneous of degree $k$, lie in $L U^{(k)} .(a, b, c)$. First note that we can obtain all elements of the form $\left(\phi_{1}, 0,0\right)$ from $Z\left(\phi_{1}\right)$, those of the form $\left(0, \phi_{2}, 0\right)$ from $Y(\beta)$ where $\partial \beta / \partial x=\phi_{2}$. Finally by choosing a suitable $\alpha$ we can obtain any term $\left(0,0, \phi_{3}\right)$ from $X(\alpha)$ provided $\phi_{3}$ is divisible by $y$. So we need to show that $\left(0,0, x^{k}\right)$ is in the "tangent space" modulo terms of degree $k+1$. The vector $W(\alpha)=Y(\alpha)-2 Z\left(\alpha_{y}\right)$ will be particularly useful here since it has a zero in the first component.

For $k=3$ we use the following vectors in the 3 -jet space:

$$
\begin{aligned}
w_{1}=X\left(x^{2}\right) & =-\left(0,0,4 x y+4 \lambda x^{3}\right), \\
w_{2}=W\left(x y+1 / 3 \lambda x^{3}\right) & =\left(0, y+\lambda x^{2}, x y+5 / 3 \lambda x^{3}\right), \\
w_{3}=X(y) & =-\left(0, y+\lambda x^{2}, 0\right) .
\end{aligned}
$$

We have $w_{2}+w_{3}=\left(0,0, x y+5 / 3 \lambda x^{3}\right)$. Using this vector and $w_{1}$ we see that $\left(0,0, x^{3}\right)$ is in the tangent space provided $\lambda \neq 0$.

For $k>3$, we distinguish two cases depending on whether $k$ is odd or even. The crucial observation is that if we assign $x$ weight 1 , and $y$ weight 2 then the operators $X$, $Y$ and $Z$ preserve weight.

For $k=2 l$ the vectors

$$
\begin{aligned}
w_{i} & =X\left(y^{i} x^{2(l-i)-1}\right), & & 0 \leq i \leq l-1, \\
w_{l+i} & =W\left(y^{i} x^{2(l-i)}\right), & & 0 \leq i \leq l,
\end{aligned}
$$

belong to the vector space of dimension $k+1$ generated by monomials of the form $\phi_{2} e_{2}$, $\phi_{3} e_{3}$ with degree $\phi_{2}=k-1$ and degree $\phi_{3}=k$. These vectors then yield a $k+1$ by $k+1$ matrix. The determinant of this matrix is a non-zero polynomial of degree $l$ in $\lambda$. (One can explicitly compute the coefficient of $\lambda$.) So it has an inverse provided $\lambda$ is not a root of this polynomial, and that leads to the elimination of $\left(0,0, x^{k}\right)$.

Similarly for $k=2 l+1$ the vectors

$$
\begin{aligned}
w_{i} & =X\left(y^{i} x^{2(l-i)}\right), & & 0 \leq i \leq l, \\
w_{l+1+i} & =W\left(y^{i} x^{2(l-i)+1}\right), & & 0 \leq i \leq l,
\end{aligned}
$$


yield a $k+1$ by $k+1$ matrix with determinant a non-identically zero polynomial of degree $l+1$ in $\lambda$. So $\left(0,0, x^{k}\right)$ can be eliminated provided $\lambda$ is not a root of the determinant.

Proposition 4.9. The BDE $d y^{2}-\left(y+\lambda x^{2}\right) d x^{2}$ is formally 2-determined.

The question of convergence of the above process remains open. In fact this result (in the smooth case) was established by Davydov in [9], and we next turn to his more geometric methods.

5. Geometrical approaches. In [9] Davydov completed a smooth classification of certain types of BDE (those covered by Proposition 4.9) at a fold point, proceeding as follows.

When the projection map $\pi$ is a fold there is a well defined involution on the surface of the equation $M$, namely that interchanging two points on the surface that project to the same image. The fixed point set of the involution naturally coincides with the criminant of the equation.

As before we label the lifted field on $M$ by $\xi$. The involution is then said to be $\xi$-good at a zero of $\xi$, if the direction field determined by $\xi$ and its image under the involution are identical only on the fixed set of the involution, and the eigenvectors of the linear part of $\xi$ and the derivative of the involution are pairwise distinct.

It is clear that the classification of the integral curves of a BDE at a fold point is equivalent to that of reducing direction fields together with $\xi$-good involutions on $M$ to normal forms. Suppose that we have a direction field which is $C^{\infty}$-equivalent to one of the three forms given at the beginning of Section 4 .

THEOREM 5.1 [9]. The germs at zero of the direction field $\xi$ and of a $\xi$-good involution can, by a $C^{\infty}$-diffeomorphism of the plane, be simultaneously reduced to the germs at zero of the direction field $\xi_{1}$ (respectively $\xi_{2}, \xi_{3}$ ) (as in Section 4) and the involution $\theta_{1}$ (respectively $\left.\theta_{1}, \theta_{2}\right)$, where

$$
\begin{aligned}
& \theta_{1}(x, y)=((\alpha+1) x-2 \alpha y, 2 x-(\alpha+1) y) /(\alpha-1), \\
& \theta_{2}(x, y)=(x-2 y / \alpha,-y) .
\end{aligned}
$$

One can eliminate the modulus $\alpha$ in the normal forms if allowing topological changes of coordinates. Thus,
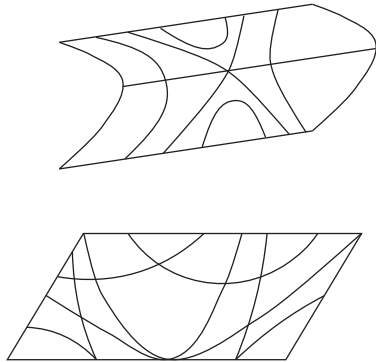

Saddle
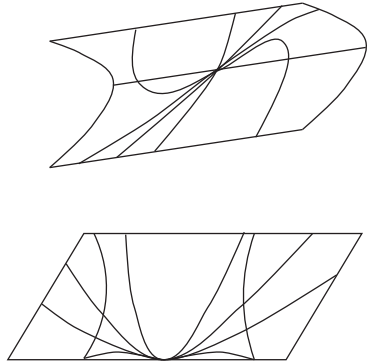

Node
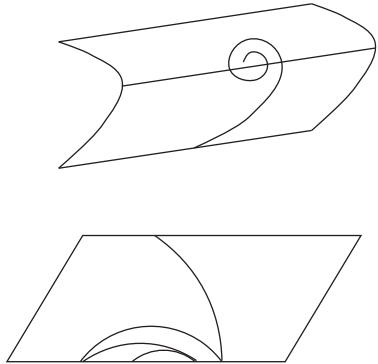

Focus

Fig. 2. Solution curves at a regular singular point 
ThEOREM 5.2 [9]. The germs at zero of the direction field $\xi$ and of a $\xi$-good involution can be simultaneously reduced by a homeomorphism of the plane to the germs at zero of the direction field $\xi_{1}$ (respectively $\xi_{2}, \xi_{3}$ ) and the involution $\theta_{1}$ (respectively $\theta_{1}, \theta_{2}$ ) for $\alpha=-2$ (respectively, $\alpha=2, \alpha=1)$. (See Figure 2.)

These three forms are referred to as the well folded node, saddle and focus respectively. This classification has applications to a number of the examples in the introduction, for which we refer you to [9]. In particular the configuration of asymptotic lines on the surface $z=y^{2}+x^{2} y+A X^{4}+O(5)$ near the origin is diffeomorphic to a well folded saddle, node or focus for $A<1 / 4,1 / 4<A<25 / 96,25 / 96<A$ respectively.

6. BDEs with discriminant of Morse type. In this final section we are concerned with BDE's of the form

$$
a(x, y) d y^{2}+2 b(x, y) d x d y+c(x, y) d x^{2}=0
$$

where $a, b, c$ are smooth functions all vanishing at $(0,0)$. As usual this equation defines a pair of directions at points of the plane $(x, y)$ where $b^{2}-a c>0$. The two directions coincide on the set $\Delta=\left\{(x, y): b^{2}-a c=0\right\}$, the discriminant of the equation, and there are no directions at points where this expression is negative. Of course the discriminant is no longer smooth in this case. We ask instead that the function $b^{2}-a c$ has a singularity of Morse type at the origin; in other words after a change of co-ordinates it can be reduced to a sum or difference of squares. We shall see below that this condition is natural as it is equivalent to the surface

$$
\left\{(x, y,[p, q]) \in \mathbb{R}^{2} \times \mathbb{R} P^{1}: a p^{2}+2 b p q+c q^{2}=0\right\}
$$

associated to the equation being smooth.

Indeed this surface is then a cylinder with the whole centre circle, which we refer to as the exceptional fibre, over the origin. We take an affine chart of $\mathbb{R}^{2} \times \mathbb{R} P^{1}$, by setting $q=1$, and consider locally in $\mathbb{R}^{3}$ the double cover surface

$$
M=\left\{(x, y, z): a p^{2}+2 b p+c=0\right\} .
$$

We shall denote the function $a p^{2}+2 b p+c$ by $F$ and write

$$
\begin{aligned}
& a=a_{1} x+b_{1} y+O(2), \\
& b=b_{1} x+b_{2} y+O(2), \\
& c=c_{1} x+c_{2} y+O(2) .
\end{aligned}
$$

Proposition 6.1 [6]. (1) The surface $M$ is smooth in a neighbourhood of $0 \times \mathbb{R} P^{1}$ if and only if the discriminant function $b^{2}-$ ac has a Morse singularity.

(2) The natural projection $\pi: M \rightarrow \mathbb{R}^{2}$ given by $(x, y, p) \mapsto(x, y)$ is a local diffeomorphism away from $\pi^{-1}(\Delta)$.

Proposition 6.2 [6]. (1) The vector field

$$
\xi=F_{p} \partial / \partial x+p F_{p} \partial / \partial y-\left(F_{x}+p F_{y}\right) \partial / \partial p
$$

is a suitable lift on $M$ of the bivalued fields in $\mathbb{R}^{2}$. (As we have seen before.) 
(2) The zeros of the vector field $\xi$ occur generically on the exceptional fibre $0 \times \mathbb{R} P^{1}$. There are in general one or three zeros given by the roots of the cubic

$$
\phi(p)=a_{2} p^{3}+\left(2 b_{2}+a_{1}\right) p^{2}+\left(2 b_{1}+c_{2}\right) p+c_{1} .
$$

The zeros are of type node or saddle.

The exceptional fibre is an integral curve passing through the zeros of $\xi$. This explains why there are no focus singularities.

The formal reduction of the BDE is dealt with in [6]. We can reduce in general the linear part of the BDE to the case where $a= \pm c=y$. (We cannot reduce the linear part $b_{1} x+b_{2} y$ of the function $b$.) We then proceed as in the previous section to annihilate all high order terms in $a$ and $c$. The matrix $M_{k}$, at the $k$-step of this procedure depends only on $b_{1}, b_{2}$ and $k$. The pair $\left(b_{1}, b_{2}\right)$ is called resonant if there exists an integer $k \geq 2$ such that the matrix $M_{k}$ is singular.

Proposition $6.3[6]$. If the pair $\left(b_{1}, b_{2}\right)$ is non-resonant, the BDE $(2)$ can be reduced by a formal diffeomorphism to

$$
y d y^{2}+2\left(b_{1} x+b_{2} y+b(x, y)\right) d x d y \pm y d x^{2}=0
$$

with $b$ a formal power series with no constant or linear terms.

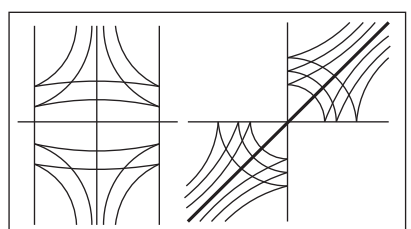

1 Saddle

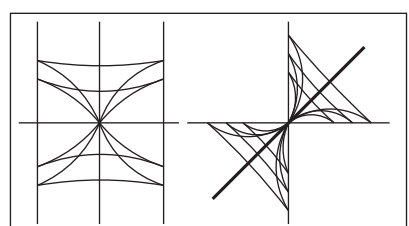

1 Node
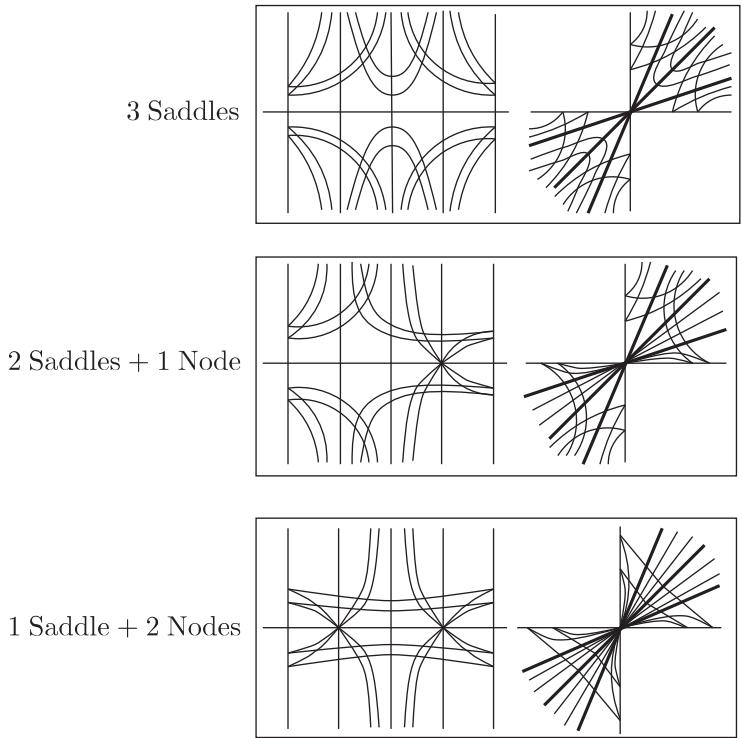

Fig. 3. Integral curves of BDE's of type II, on the Möbius band left and on the plane right 
Note that any BDE of the form $(a, b,-a)$ has its integral curves mutually orthogonal (the (-) case above. The convergence of the above process is still an open question. We obtain topological normal forms using an alternative method.

6.1. Topological normal forms. The case where the BDE defines a pair of orthogonal directions (i.e. $a=-c$ ) was treated in [5]. The argument used there is extended in [6] to deal with the general case. We have started by lifting the bivalued vector field to a single valued field on a cylinder $M$, with the centre circle of the cylinder corresponding to the origin. However we need to work with the bivalued fields in the plane, so we need to identify pairs of points on the cylinder which correspond to the same point on the plane. For the case $a=-c$ treated in [5] the identification can be made quite explicit, it simply sends $(x, y, p)$ to $(x, y,-1 / p)$. In general, we show [6] that the involution on $M$ that interchanges pairs of points corresponding to the same image in the plane, which is well defined away from the exceptional fibre, and extends smoothly across this fibre. (We used Riemann's extension theorem to prove this assertion, so we need to restrict to the case where $a, b, c$ are analytic.) Consequently we reduce to the classification of certain bivalued vector fields on the quotient space, a Möbius band $M^{\prime}$.

If the discriminant is an isolated point, that is the function $b^{2}-a c$ has a local maximum or minimum, then as in [5], the new bivalued field is defined in a neighbourhood of the whole central line of the Möbius band. In the case where the discriminant is a node the identification map has, in addition to the central line, two lines of fixed points. So we need to construct a model of the integral curves along these critical lines. This can be done using the machinery of singularity theory.

Away from these lines the argument is as in [5] for both cases. Suppose for simplicity that the lifted field $\xi$ on $M$ has only one zero. Then we can construct a homeomorphism between a neighbourhood of the zero of the bivalued field on $M^{\prime}$ and one on our model, and this homeomorphism is then extended to the intervening strips. If $\xi$ has three zeros we fix up the homeomorphism on a neighbourhood of the zeros to start with and then extend again on the intervening strips.

We obtain the following result, where we assume that the discriminant is of Morse type, the cubic $\phi$ in Proposition 6.2 has no double roots and the zeros of the field $\xi$ are normal (i.e. can be reduced to the normal forms in Section 4).

TheOREM 6.4 [6]. There is a germ of homeomorphism $h: \mathbb{R}^{2}, 0 \longrightarrow \mathbb{R}^{2}, 0$ taking the integral curves of the BDE (2) to the integral curves of one of the normal forms:

I. The discriminant is an isolated point (Theorem 0.1, [5]) (see Figure 1):
(a) Lemon $\quad y d y^{2}+2 x d x d y-y d x^{2}=0$
(b) Star $\quad y d y^{2}-2 x d x d y-y d x^{2}=0$
(c) Monstar $y d y^{2}+\frac{1}{2} x d x d y-y d x^{2}=0$.

II. The discriminant is a crossing of two smooth curves (see Figure 3 ):
(a) 1 saddle $y d y^{2}+2 x d x d y+y d x^{2}=0$
(b) 1 node $\quad y d y^{2}-\frac{1}{2} x d x d y+y d x^{2}=0$
(c) 3 saddles $y d y^{2}-4 x d x d y+y d x^{2}=0$ 


$$
\begin{aligned}
& \text { (d) } 2 \text { saddles }+1 \text { node } y d y^{2}+2(y-x) d x d y+y d x^{2}=0 \\
& \text { (e) } 1 \text { saddle }+2 \text { nodes } y d y^{2}-\frac{4}{3} x d x d y+y d x^{2}=0 .
\end{aligned}
$$

The type is determined by the 1-jet of the functions a, b, c. Moreover this homeomorphism is a diffeomorphism away from certain singular directions through the origin.

There are a number of applications, for example to principal curves near an umbilic, asymptotic curves at a flat umbilic, asymptotic curves on a cross-cap, asymptotic curves on a surface in 4-space. See for example [6], [13].

Acknowledgement. We are much indebted to Dr Richard Morris, whose computer package 'Liverpool Surface Modelling Package' produced some of the configurations reproduced here.

\section{References}

[1] V. I. Arnold, Geometrical Methods in the Theory of Ordinary Differential Equations, Springer, Berlin, 1983.

[2] Th. Brocker and L. C. Lander, Differentiable Germs and Catastrophes, London Math. Soc. Lecture Note Ser. 17, Cambridge University Press, 1975.

[3] J. W. Bruce, A note on first order differential equations of degree greater than one and wavefront evolution, Bull. London Math. Soc. 16 (1984), 139-144.

[4] J. W. Bruce, A. A. du Plessis and C. T. C. Wall, Determinacy and unipotency, Invent. Math. 88 (1987), 521-554.

[5] J. W. Bruce and D. Fidal, On binary differential equations and umbilics, Proc. Roy. Soc. Edinburgh 111A (1989), 147-168.

[6] J. W. Bruce and F. Tari, On binary differential equations, Nonlinearity 8 (1995), 255271.

[7] L. Dara, Singularités génériques des équations différentielles multiformes, Bol. Soc. Brasil. Math. 6 (1975) 95-128.

[8] G. Darboux, Leçons sur la théorie générale des surfaces, Vol. 4, Gauthier-Villars, Paris, 1896.

[9] A. A. Davydov, Normal forms of differential equations unresolved with respect to derivatives in a neighbourhood of a singular point, Functional Anal. Appl. 19 (1985), 1-10.

[10] C. Gutierrez and J. Sotomayor, Structurally stable configurations of lines of principal curvature, Astérisque (1982), 98-99.

[11] - - - Lines of curvature and umbilical points on surfaces, 18 Colóquio Brasileiro de Matemática, Instituto de Matemática Pura e Aplicada, Brasil, 1991.

[12] A. D. Myshkis, Differential inequalities with locally bounded derivatives, Zap. Mekh.Mat. F-ta Khar'kovskigo Mat. O-va 30 (1964), 152-163.

[13] J. M. West, Ph. D. Thesis, Liverpool University, 1995.

[14] H. Whitney, On singularities of mappings of Euclidean spaces I, Mappings from the plane to the plane, Ann. of Math. 62 (1955), 374-410. 